\title{
Dopant-Free $\pi$-Conjugated Hole Transport Materials for Highly Stable and Efficient Perovskite Solar Cells
}

\author{
Zhifeng Deng ${ }^{1,2 \star t}$, Shuaiwei Cui ${ }^{3 \dagger}$, Kaichang Kou ${ }^{1}$, Dongxu Liang ${ }^{3}$, Xin Shi ${ }^{2}$ and \\ Jinhui Liu ${ }^{3 *}$
}

${ }^{1}$ School of Chemistry and Chemical Engineering, Northwestern Polytechnical University (NWPU), Xi'an, China, ${ }^{2}$ National and Local Joint Engineering Laboratory for Slag Comprehensive Utilization and Environmental Technology, School of Materials Science and Engineering, Shaanxi University of Technology, Hanzhong, China, ${ }^{3}$ Key Laboratory of Rubber-Plastic of Ministry of Education (QUST), School of Polymer Science and Engineering, Qingdao University of Science and Technology, Qingdao, China

Current high-efficiency hybrid perovskite solar cells (PSCs) have been fabricated with doped hole transfer material (HTM), which has shown short-term stability. Doping applied in HTMs for PSCs can enhance the hole mobility and PSCs' power conversion

OPEN ACCESS

Edited by:

Qixin Zhou,

University of Akron, United States

Reviewed by:

Cheng Zhang,

National Renewable Energy Laboratory (DOE), United States

Qianqian Su,

Shanghai University, China

*Correspondence:

Zhifeng Deng

dengzf@snut.edu.cn

Jinhui Liu

liujinhui7@163.com

†These authors have contributed equally to this work

Specialty section: This article was submitted to

Organic Chemistry,

a section of the journal

Frontiers in Chemistry

Received: 05 February 2021 Accepted: 25 February 2021

Published: 18 March 2021

Citation:

Deng Z, Cui S, Kou K, Liang D, Shi X and Liu J (2021) Dopant-Free $\pi$-Conjugated Hole Transport Materials for Highly Stable and Efficient Perovskite Solar Cells.

Front. Chem. 9:664504. doi: 10.3389/fchem.2021.664504 efficiency, while the stability of PSCs will be significantly decreased due to inherent hygroscopic properties and chemical incompatibility. Development of dopant-free HTM with high hole mobility is a challenge and of utmost importance. In this review, a series of selected and typical $\pi$-conjugated dopant-free hole transport materials, mainly regarding small molecules, are reviewed, which could consequently help to further design high-performance dopant-free HTMs. In addition, an outline of the molecular design concept and also the perspective of ideal dopant-free HTMs were explored.

Keywords: $\pi$-conjugated materials, perovskite solar cells, hole transfer material, dopant-free, molecular design

\section{INTRODUCTION}

Currently, the state-of-the-art perovskite solar cells with conventional $n$-i- $p$ structures utilize 2,2',7,7'-tetrakis( $N, N^{\prime}$-di-p-methoxyphenlamine)-9,9-spirobifluorene (spiro-OMeTAD) or poly-triarylamine (PTTA) as HTMs. However, the HTMs like spiro-OMeTAD and PTAA suffer from low mobility $\left(<1 \times 10^{-5} \mathrm{~cm}^{2} \mathrm{~V}^{-1} \mathrm{~s}^{-1}\right)$ and limited conductivity $(<3 \times$ $10^{-7} \mathrm{~S} \mathrm{~cm}^{-1}$ ) (Vivo et al., 2017). These materials thus need hazardous dopants to increase the hole mobility and conductivity; these can include such dopants as bis(trifluoromethane sulfonyl)imide lithium salt (LiTFSI), which may also facilitate device degradation due to the sophisticated oxidation process associated with undesired ion migration and chemical interaction with underlying perovskite layer ( $\mathrm{Yu}$ and Sun, 2015; Abi Ghanem et al., 2019). In addition, such dopants would make the device prone to hygroscopicity. Considering their drawbacks, over the last few years, a wide range of novel HTMs have been proposed as alternatives by the state-of-the-art organic $\pi$-conjugated small molecule and polymer; however, only a few candidates have reached similar initial performance compared to the state-of-the-art HTMs. Unfortunately, those candidates still require high amounts of dopants to operate ( $\mathrm{Yu}$ and Sun, 2015; Vivo et al., 2017). Even though a few operational dopant-free materials could be obtained, the power conversion efficiency (PCE) still lacks behind the state-of-the-art HTMs (Huang et al., 2016; Liu et al., 2016; Yun et al., 2016; Zhao et al., 2016; Lee et al., 2017). Normally, HTMs would not require an additional doping process if they exhibit hole mobility up to $10^{-4}-10^{-3} \mathrm{~cm}^{2} \mathrm{~V}^{-1} \mathrm{~s}^{-1}$ and have matched energy levers. Therefore, the development of 
high-performance dopant-free HTMs is highly desirable and of utmost importance. Molecular design strategies, such as large $\pi$-conjugation and strong $\pi-\pi$ stacking (Paek et al., 2017), strong planarity (Yun et al., 2016), surface passivation (Cao et al., 2015), introducing functional groups, and novel chemical structures, have been considered to be the most feasible and effective methods mainly used for designing dopant-free HTMs that possess better intermolecular interactions to ensure sufficient hole mobility. In this article, dopant-free $\pi$-conjugated small molecules applied to PSCs are reviewed.

\section{LARGE $\pi$-CONJUGATION AND STRONG $\pi-\pi$ STACKING}

Dopant-free HTMs are divided into small molecules and polymers. Compared to polymers, small organic molecular HTMs have received great attention due to their high efficiency and simple synthetic schemes (Zhang et al., 2017a). Simultaneously, they were also shown to have better intramolecular hole transfer mobility due to better packing. In general, the molecular structure of organic HTMs consists of peripheral donors and central cores, such as pyrene (Ge et al., 2018), fluorene (Rakstys et al., 2017b), carbazole (Chen et al., 2017), benzothiophene (Zimmermann et al., 2017), and indacenodithienothiophene (Liu et al., 2017). The studies on dopant-free organic HTM are quite rare, among which most reported molecules contain thiophene or thiophene derivatives as the donor units or $\pi$-conjugated bridge. Very recently, $\mathrm{K}$. Nazeeruddin and co-authors using rigid quinolizino acridine (FA), which is widely used in molecular semiconductors due to its planarity and strong $\pi-\pi$ stacking, as the central donor core, tri-thiophene as the $\pi$-conjugated bridge, and malenonitrile as the strongest electron acceptor to stabilize the highest occupied molecular orbital (HOMO) energy level, which built a branch oligomer (S1, Figure 1) and showed PCE of $18.9 \%$ and a maximum power output that was collected after $1300 \mathrm{~h}$ that remained at $65 \%$ of its initial value (Paek et al., 2017). In addition, this group introduced thiophene or tri-thiophene as branches into the same core and synthesized two oligomers (S2 and S3, Figure 1), which were also used as HTMs to fabricate PSCs (Rakstys et al., 2017a). The results showed that $\pi$-extension would significantly enhance the PCE of the PSCs from 8.88 to $19.03 \%$.

\section{STRONG PLANARITY}

Except for $\pi$-conjugation extension, the planarity of a molecular backbone is crucial for the molecular design concept of high-performance dopant-free HTMs. J. Son and co-authors introduced fluorine atoms to the molecules for adjusting the planarity of the backbone and the molecular packing through sulfur-fluorine interactions, which significantly increased the hole mobility and the PCE from $8.95 \%$ (S4) to $14.45 \%$ (S5) (Yun et al., 2016). Very recently, through a comprehensive study on the deliberate molecular design and modifications of electron donors, correlations between the planarity of HTMs backbone and performance of PSC were reported by Liang's group (Li et al., 2020). In this work, the dithieno[3,2-b:2',3'-d]pyrrole (DTP) both end substituted by the twisted triphenylamine as the donor (S6) or the capped-by-planar- $N$-phenyl-carbazole donor were synthesized (S7). We used two small molecules, S6 and S7, as dopant-free HTMs to fabricate PSCs, which showed PCEs with $11.59 \%$ (S6) and $18.29 \%$ (S7). The chemical structure between triphenylamine and the $N$-phenyl-carazole are quite similar except for an additional chemical bond connecting between the two phenyl rings of the triphenylamine; however, the PCE of the S7-based device showed a value almost $60 \%$ higher than the one based on S6. The significantly different performance between these two small molecule-based PSCs could be ascribed to S7 with an improved planarity backbone, resulting in a good hole transfer ability.

\section{SURFACE PASSIVATION (INTERFACIAL INTERACTION)}

In a photovoltaic device, after light absorption, the generated charge carriers (electron/hole) need to be transported through the perovskite layer and collected at the adjacent charge selective interfaces (Zhang et al., 2017b). Each of these steps plays a key role in the high performance of PSCs. Among them, the quite important (and mostly neglected) part is the hole transfer between the interface of HTM and the perovskite layer. To the best of our knowledge, most reported articles focus on the modification of the HTM's HOMO/LUMO energy levels but not on the inter-layer interaction ( $\mathrm{Yu}$ and Sun, 2015; Vivo et al., 2017). Compatible HOMO/LUMO energy levels for HTMs will be beneficial for the hole transfer and to block the electron transfer from the perovskite layer to the HTM layer to enable the maximum achievable open-circuit voltage. The good HTM should not only have a compatible HOMO energy level but should also provide intimate interlayer interaction since the interface interaction would help to rapidly extract holes from the perovskite layer and transfer them to the HTM layer, resulting in a low energy loss and reduced recombination of charge carriers at the interface. Zheng's group reported that a thiolated nanographene perthiolated trisulfurannulated hexa-peri-hexabenzocoronene (S8, Figure 2), as the HTM in the pristine form in PSCs (Cao et al., 2015). The thiol groups at the periphery form $\mathrm{Pb}-\mathrm{S}$ coordination-bonds at the interface between perovskite and HTM, which was investigated by the infrared spectra. The tight binding of S8 helps to rapidly extract charge from perovskite, resulting in a low energy loss at the interface and PCE of $12.8 \%$. The performance is readily improved by doping with graphene sheets into trisulfurannulated hexa-peri-hexabenzocoronene (TSHBC), which formed a novel functionalized nanographene that could enhance the hole transporting property within HTM. In addition, M. Zhan and co-workers reported on the introduction of an interaction layer between the perovskite and HTM layers, which could act as Lewis bases and interact with $\mathrm{Pb}$ atoms to form trap states that greatly passivate the defects on the surface of the perovskite layer (Zhang et al., 2017b). This technique could 

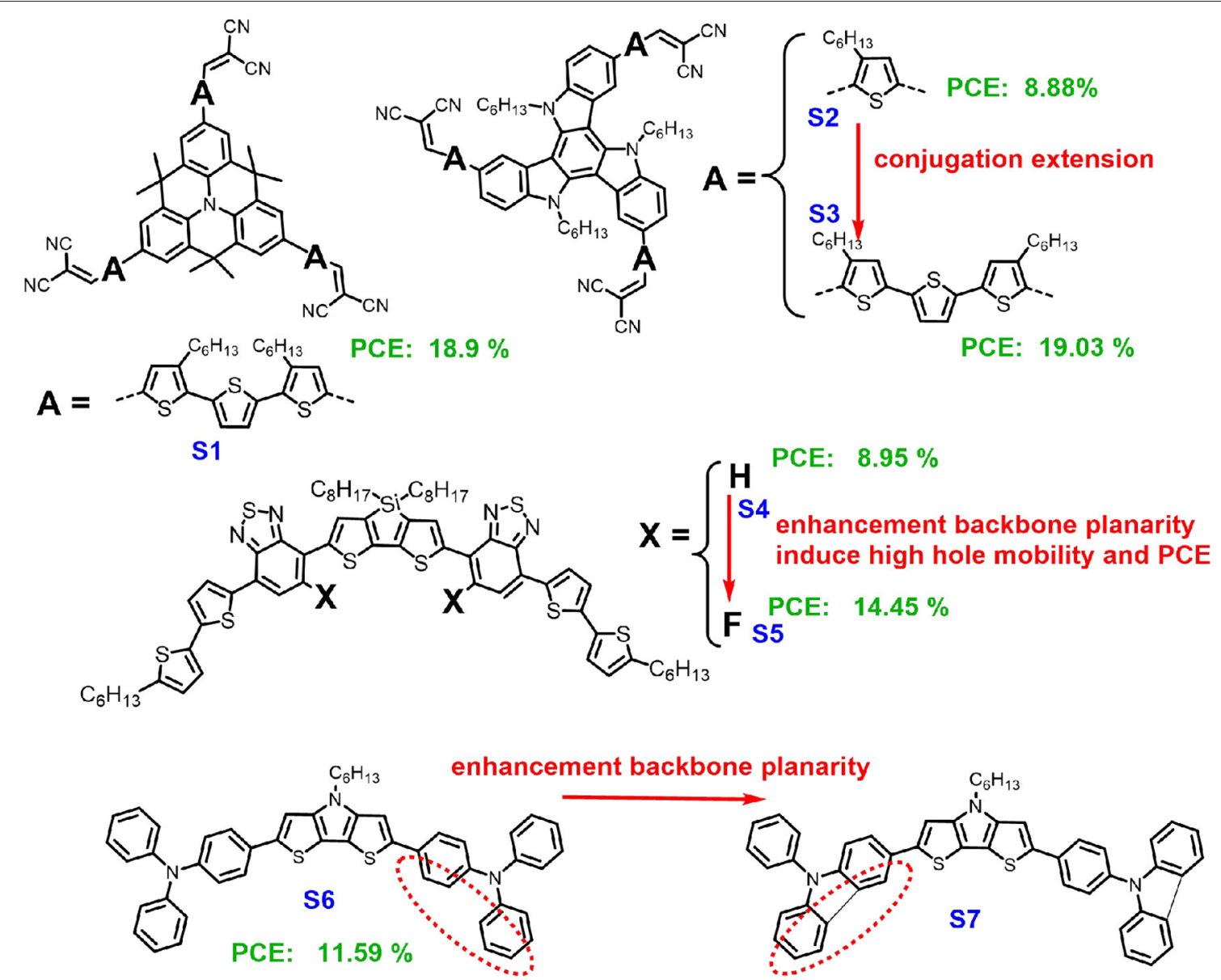

PCE: $18.29 \%$

FIGURE 1 | Chemical structures of small molecules S1-S7 for dopant-free HTMs.

significantly enhance the efficiency and stability of the PSCs. Except for sulfur atoms, silicon and nitrogen atoms can also provide lone pair electrons to form $\mathrm{Pb}-\mathrm{N}$ or $\mathrm{Pb}-\mathrm{Si}$ coordinationbonds through the formation of Lewis adduct between the under-coordinated $\mathrm{Pb}$ atoms at the HTM-perovskite interface. A deeper understanding of the relationship between $\mathrm{Pb}-\mathrm{S} / \mathrm{Pb}$ $\mathrm{N} / \mathrm{Pb}-\mathrm{S}$ interaction at perovskite/HTM interface and the PSC's performance should be established in the near future.

\section{INTRODUCING FUNCTIONAL GROUP}

Research into organic electronics revealed that not only the composition of the organic material but also subtle changes in the material structure (the way how the molecules are stacked) can noticeably alter its bulk properties (Yao et al., 2016). One of the reasons is that the charge transport in conjugated materials is strongly affected by their structures. For totally disordered organic small molecular films, charge mobility is low, while mobility significantly increases if the materials exhibit selfassembling properties that can be exploited to generate ordered structures (Zhang et al., 2018, 2020). Jian et al. designed and synthesized tetrathia-fulvalene derivative (S9, Figure 2) and used it as dopant-free HTM, which showed a PCE of $11.03 \%$ in 2014, fill factor (FF) of $64 \%$, and open-circuit voltage $\left(V_{\mathrm{oc}}\right)$ of $0.86 \mathrm{~V}$ (Liu et al., 2014). A few years later, Islam's group introduced two amide units into these small molecules (S10) (Kaneko et al., 2019). In S10, intermolecular hydrogen bonds could be formed between amino and the carbonyl groups of the neighboring two molecules in the solid states. Using this small molecule to fabricate the PSCs, a PCE of $14.5 \%$ with the $V_{\text {oc }}$ of $1.11 \mathrm{~V}$ and the FF of $66 \%$ were achieved. The increased PSCs performance could be ascribed to the following facts: (i) hydrogen bonding formation between the adjacent molecules, which could improve the inter-molecular hole transfer mobility; and (ii) the exiting multi-nitrogen and oxygen atoms could form surface passivation between the HTMs layer and the perovskite layer, which is beneficial for the hole transfer between the two layers.

\section{MATERIALS WITH NOVEL CHEMICAL STRUCTURES}

For high-performance dopant-free HTMs for PSCs, the development of novel chemical structures are important. For commercial applications, it is a challenge to find suitable and 


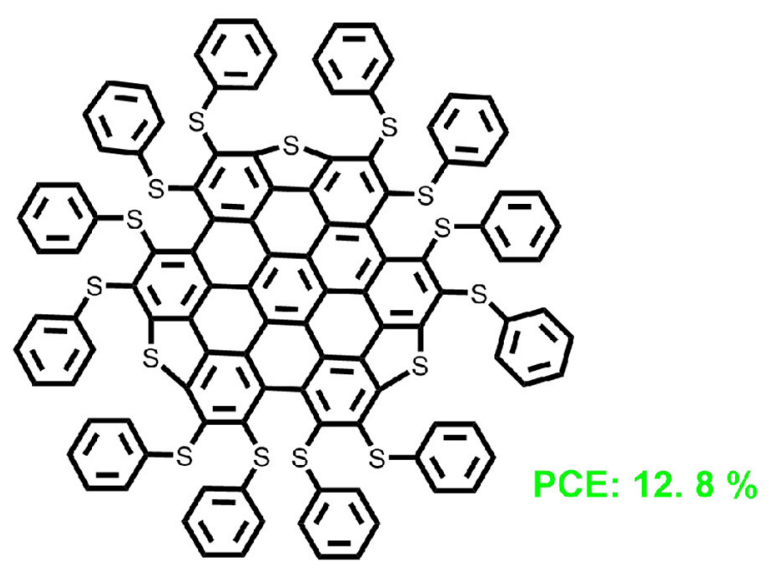

58

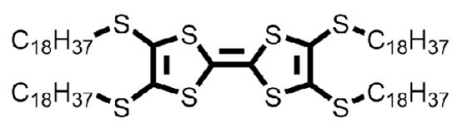

S9

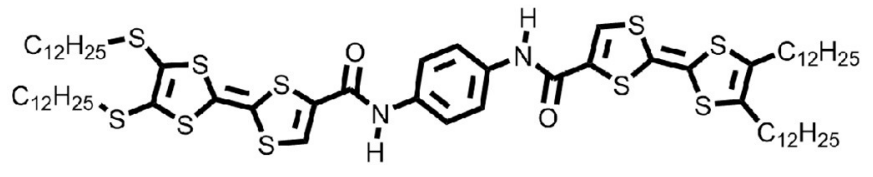

S10
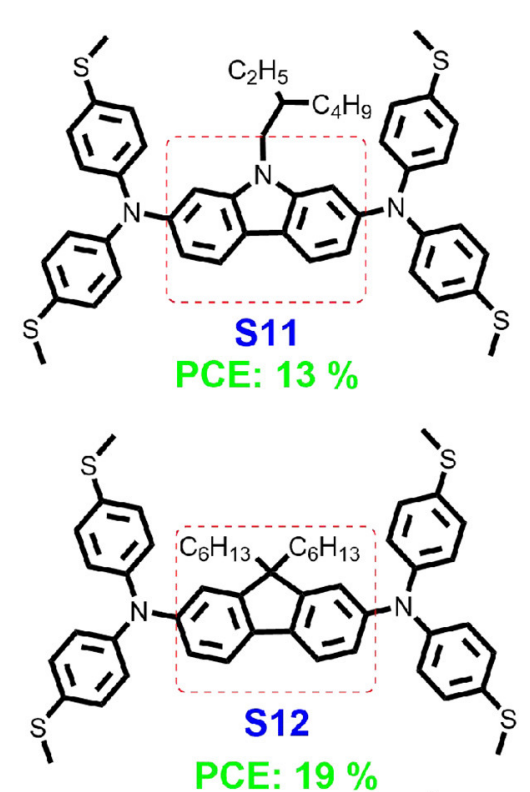

PCE: $11 \%$

PCE: $14.5 \%$

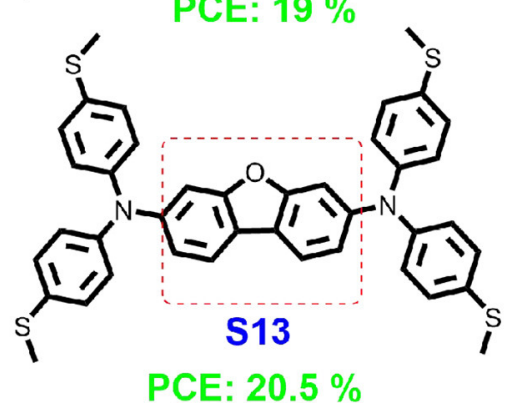

FIGURE 2 | Chemical structures of small molecules S8-S13 for dopant-free HTMs.

low-cost HTMs in PSCs. In 2017, Ding's group designed a novel HTM based on carbazole and $N, N$-di- $p$-methylthiophenylamine, which is named S11 (Figure 2) (Xu et al., 2017). The dopant-free S11-based planar $p$ - $i$ - $n$ perovskite solar cells exhibit a high PCE of $13.05 \%$ with a $V_{\text {oc }}$ of $1.03 \mathrm{~V}$ and FF of $58.23 \%$. Later, the same group modified the structure using 9,9-dihexyl-9H-fluorene instead of the carbazole core obtained S12 (Zhang et al., 2019). Using S12 to fabricated the PSCs showed significantly improved performance with a higher PCE of $19.06 \%, V_{\text {oc }}$ of $1.07 \mathrm{~V}$, and FF of $79.27 \%$. In 2020, the authors further optimized the HTMs structures and changed the core into dibenzo[b,d]furan, resulting in S13. The performance of the PSC-based S13 even further improved with a PCE up to $20.51 \%, V_{\text {oc }}$ of $1.07 \mathrm{~V}$, and FF of $80.48 \%$ (Quan et al., 2020). This investigation indicates that the molecular design is quite important for high-performance HTMs, and developing novel structures is thus crucial.

\section{CONCLUSIONS AND OUTLOOK}

Research regarding HTMs for PSCs is crucial and could significantly improve the performance of perovskite solar cell devices in terms of power conversion efficiency, open-circuit voltage, fill factor, etc. Furthermore, due to the high conductivity and hole transfer mobility, HTMs do not need dopant in the HTM layer. The cost of the HTMs is thus decreased; meanwhile, the lifetimes of the PSCs are extended. This review revealed that the ideal HTMs of small molecules could be achieved with high conductivity and hole transfer mobility with low cost and suitable energy levels. To obtain such ideal dopant-free HTMs, several molecular design concepts are summarized in this review, such as enlarging the $\pi$-conjugation system, increasing the planarity of molecular backbone, introducing the functional atoms/groups to achieve interface interaction between the HTMs layer and the perovskite layer, and, resulting in self-assembly within the HTMs layer, the development molecules with novel chemical structures. HTMs play a key role in the high performance of PSCs. Future research should focus on the combination of part or all of the abovementioned design concepts in a single molecule to develop desirable HTMs.

\section{AUTHOR CONTRIBUTIONS}

ZD and SC prepared the manuscript. DL and XS helped to prepare the references and revise the manuscript. KK revised the manuscript. ZD and JL supervised the whole work. All authors discussed and commented on the paper. 


\section{FUNDING}

This study was financially supported by Research Projects of Shaanxi University of Technology (SLGPT2019KF01-01, SLG1901).

\section{REFERENCES}

Abi Ghanem, M., Liang, X., Lydon, B., Potocsnak, L., et al. (2019). Wrinkles: wrinkles riding waves in soft layered materials. Adv. Mater. Interfaces 6:1970004. doi: 10.1002/admi.201970004

Cao, J., Liu, Y.-M., Jing, X. J., Yin, J., Li, J., Xu, B., et al. (2015). Welldefined thiolated nanographene as hole-transporting material for efficient and stable perovskite solar cells. J. Am. Chem. Soc. 137, 10914-10917. doi: 10.1021/jacs.5b06493

Chen, Z. L., Li, H., Zheng, X. L., Zhang, Q., Li, Z. F., et al. (2017). Low-cost carbazole-based hole-transport material for highly efficient perovskite solar cells. ChemSusChem 10, 3111-3117. doi: 10.1002/cssc.201700678

Ge, Q.-Q., Shao, J.-Y., Ding, J., Deng, L.-Y., and Zhou, W.-K. (2018). A two-dimensional hole-transporting material for high-performance perovskite solar cells with 20\% average efficiency. Angew. Chem. 130, 11125-11131. doi: 10.1002/ange.201806392

Huang, C. Y., Fu, W. F., Li, C.-Z., Zhang, Z. Q., Qiu, W. M., Shi, M. M., et al. (2016). Dopant-free hole-transporting material with a $\mathrm{C}_{3 \mathrm{~h}}$ symmetrical truxene core for highly efficient perovskite solar cells. J. Am. Chem. Soc. 138, 2528-2531. doi: 10.1021/jacs.6b00039

Kaneko, R., Chowdhury, T. H., Sugawa, K., Lee, J.-J., Otsuki, J., and Islam, A. (2019). Electro-active nanofibers of a tetrathiafulvalene derivative with amide hydrogen bonds as a dopant-free hole transport material for perovskite solar cells. Solar Energy 194, 248-253. doi: 10.1016/j.solener.2019.10.078

Lee, J., Byranvand, M. M., Kang, G., Son, S. Y., Song, S., Kim, G.-W., et al. (2017). Green-solvent-processable, dopant-free hole-transporting materials for robust and efficient perovskite solar cells. J. Am. Chem. Soc. 139, 12175-12181. doi: 10.1021/jacs.7b04949

Li, M. Y., Wu, J. H., Wang, G. G., Wu, B. X., Sun, Z., et al. (2020). The donor-dependent methoxy effects on the performance of holetransporting materials for perovskite solar cells. J. Energy Chem. 47, 10-17. doi: 10.1016/j.jechem.2019.11.017

Liu, J., Wu, Y., Qin, C., Yang, X., Yasuda, T., Islam, A., et al. (2014). Dopant-free hole-transporting material for efficient and stable perovskite solar cells. Energy Environ. Sci. 7, 2963-2967. doi: 10.1039/C4EE01589D

Liu, X. Y., Zheng, X. L., Wang, Y. L., Chen, Z. L., Yao, F., et al. (2017). Dopantfree hole-transport materials based on methoxytriphenylamine-substituted indacenodithienothiophene for solution-processed perovskite solar cells. ChemSusChem 10, 2833-2838. doi: 10.1002/cssc.201700197

Liu, Y. S., Hong, Z. R., Chen, Q., Chen, H. J., Chuang,W.-H., Yang, Y. M., et al. (2016). Perovskite solar cells employing dopant-free organic hole transport materials with tunable energy levels. Adv. Mater. 28, 440-446. doi: 10.1002/adma.201504293

Paek, S., Qin, P., Lee, Y. H., Cho, K. T., Gao, P., Grancini, G., et al. (2017). Dopantfree hole-transporting materials for stable and ffficient perovskite solar cells. Adv. Mater. 29:1606555. doi: 10.1002/adma.201606555

Quan, S., Jing, Z., Chen, Q. Y., Wang, Y. K., Zhou, Y., Song, B., et al. (2020). High-efficiency planar $p-i-n$ perovskite solar cells based on dopant-free dibenzo[b,d]furan-centred linear hole transporting material. J. Power Sources 449:227488. doi: 10.1016/j.jpowsour.2019.227488

Rakstys, K., Paek, S., Gao, P., Gratia, P., Marszlek, T., et al. (2017a). Molecular engineering of face-on oriented dopant-free hole transporting material for perovskite solar cells with $19 \%$ PCE. J. Mater. Chem. A. 5, 7811-7815. doi: 10.1039/C7TA01718A

Rakstys, K., Paek, S., Grancini, G., Gao, P., Jankauskas, V., Asiri, A. M., et al. (2017b). Low-cost perovskite solar cells employing dimethoxydiphenylaminesubstituted bistricyclic aromatic enes as hole transporting materials. ChemSusChem 10, 3825-3832. doi: 10.1002/cssc.201700974

\section{SUPPLEMENTARY MATERIAL}

The Supplementary Material for this article can be found online at: https://www.frontiersin.org/articles/10.3389/fchem. 2021.664504/full\#supplementary-material

Vivo, P., Salunke, J. K., and Priimagi, A. (2017). Hole-transporting materials for printable perovskite solar cells. Materials 10:1087. doi: 10.3390/ma10091087

Xu, L., Huang, P., Zhang, J., Jia, X., Ma, Z., Sun, Y., et al. (2017). N,N-Di-paramethylthiophenylamine-substituted (2-ethylhexyl)-9H-carbazole: a simple, dopant-free hole-transporting material for planar perovskite solar cells. J. Phys. Chem. C. 121, 21821-21826. doi: 10.1021/acs.jpcc.7b04469

Yao, J., Yu, C., Liu, Z., Luo, H., Yang, Y., Zhang, G., et al. (2016). Significant improvement of semiconducting performance of the diketopyrrolopyrrolequaterthiophene conjugated polymer through side-chain engineering via hydrogen-bonding. J. Am. Chem. Soc. 138, 173-185. doi: 10.1021/jacs.5b09737

$\mathrm{Yu}, \mathrm{Z}$., and Sun, L. (2015). Recent progress on hole-transporting materials for emerging organometal halide perovskite solar Cells. Adv. Energy Mater. 5:1500213. doi: 10.1002/aenm.201500213

Yun, J. H., Park, S., Heo, J. H., Yoon, S., Kang, J., et al. (2016). Enhancement of charge transport properties of small molecule semiconductors by controlling fluorine substitution and effects on photovoltaic properties of organic solar cells and perovskite solar cells. Chem. Sci. 7, 6649-6661. doi: 10.1039/C6SC02448C

Zhang, H. C., Deng, R. N., Wang, J., Li, X., Chen, Y.-M., Liu, K. W., et al. (2017a). Crystalline organic pigment-based field-effect transistors. ACS Appl. Mater. Interfaces 9, 21891-21899. doi: 10.1021/acsami.7b03170

Zhang, H. C., Li, R., Deng, Z. F., Cui, S. W., Wang, Y. H., Zheng, M., et al. (2020). $\pi$-Conjugated oligomers based on aminobenzodifuranone and diketopyrrolopyrrole. Dyes Pigm. 181:108552. doi: 10.1016/j.dyepig.2020.108552

Zhang, H. C., Liu, K. W., Wu, K. Y., Chen, Y. M., Deng, R. N., Li, $\mathrm{X}$, et al. (2018). Hydrogen-bonding-mediated solid-state self-assembled isoepindolidiones (isoEpi) crystal for organic field-effect transistor. J. Phys. Chem. C. 122, 5888-5895. doi: 10.1021/acs.jpcc.7b11992

Zhang, J., Sun, Q., Chen, Q., Wang, Y., Zhou, Y., Song, B., et al. (2019). High efficiency planar p-i-n perovskite solar cells using low-cost fluorene-based hole transporting material. Adv. Funct. Mater. 29:1900484. doi: 10.1002/adfm.201900484

Zhang, M., Wang, J., Li, L., Zheng, G., Liu, K., Qin, M., et al. (2017b). High-mobility p-type organic semiconducting interlayer enhancing efficiency and stability of perovskite solar cells. Adv. Sci. 4:1700025. doi: 10.1002/advs.201700025

Zhao, X., Zhang, F., Yi, C., Bi, D., Bi, X., Wei, P., et al. (2016). A novel one-step synthesized and dopant-free hole transport material for efficient and stable perovskite solar cells. J. Mater. Chem. A. 4, 16300-16334. doi: 10.1039/C6TA05254A

Zimmermann, I., Urieta-Mora, J., Gratia, P., Aragó, J., Grancini, G., MolinaOntoria, A., et al. (2017). High-efficiency perovskite solar cells using molecularly engineered, thiophene-rich, hole-transporting materials: influence of alkyl chain length on power conversion efficiency. Adv. Energy Mater. 7:1601674. doi: 10.1002/aenm.201601674

Conflict of Interest: The authors declare that the research was conducted in the absence of any commercial or financial relationships that could be construed as a potential conflict of interest.

The handling Editor declared a past co-authorship with one of the authors ZD.

Copyright (C) 2021 Deng, Cui, Kou, Liang, Shi and Liu. This is an open-access article distributed under the terms of the Creative Commons Attribution License (CC BY). The use, distribution or reproduction in other forums is permitted, provided the original author(s) and the copyright owner(s) are credited and that the original publication in this journal is cited, in accordance with accepted academic practice. No use, distribution or reproduction is permitted which does not comply with these terms. 